\title{
Coherent radiation from neutral molecules moving above a grating
}

\author{
Alexey Belyanin ${ }^{1,2}$, Federico Capasso ${ }^{3}$, Vitaly Kocharovsky ${ }^{1,2}$, and Vladimir Kocharovsky ${ }^{2}$ \\ ${ }^{1}$ Physics Department and Institute for Quantum Studies, \\ Texas AEM University, College Station, TX 77843-4242 \\ 2 Institute of Applied Physics, \\ Russian Academy of Science, \\ 46 Ulyanov Street, 603600 Nizhny Novgorod, Russia \\ ${ }^{3}$ Bell Laboratories, Lucent Technologies, \\ 600 Mountain Avenue, Murray Hill, NJ07974, USA
}

(Dated: November 21, 2018)

\begin{abstract}
We predict and study the quantum-electrodynamical effect of parametric self-induced excitation of a molecule moving above the dielectric or conducting medium with periodic grating. In this case the radiation reaction force modulates the molecular transition frequency which results in a parametric instability of dipole oscillations even from the level of quantum or thermal fluctuations. The present mechanism of instability of electrically neutral molecules is different from that of the well-known Smith-Purcell and transition radiation in which a moving charge and its oscillating image create an oscillating dipole. We show that parametrically excited molecular bunches can produce an easily detectable coherent radiation flux of up to a microwatt.

PACS numbers: 42.50.Gy, 42.50.Md, 33.50.-j, 32.80.Qk
\end{abstract}

Introduction. The presence of conducting or dielectric surfaces near an atom or a molecule modifies its properties in a fundamental way, changing its radiation and back reaction as well as the electromagnetic vacuum fluctuations, and giving rise to a number of interesting effects; see, e. g., 迎, 2, 3, 田, 5, 6, 7, 8, 9, 10]. In particular, the atomic energy levels and the radiative decay rate are changed. A boundary can alter also the dynamics of atomic or molecular dipole oscillations. Here we describe a new effect of the latter type.

The calculations of the level shifts for a molecule near a perfectly conducting wall can be traced back to the papers [1, 11, 12. According to subsequent works [13, 14, 15, 16] (and in accordance with fluctuationdissipation theorem), this effect is due to the modification of both electromagnetic vacuum fluctuations and radiation reaction. Note, however, that in the near zone $R \ll \lambda_{0} / \sqrt{\varepsilon_{1}}$ the influence of a boundary between two media with dielectric constants $\varepsilon_{1}$ and $\varepsilon_{2}$ on a radiating dipole is dominated by the radiation reaction force, while the contribution from vacuum fluctuations of the electromagnetic field is negligible. This result has been obtained in [15] using the ideas of separating the selfaction and vacuum-fluctuation terms developed in [14; see 3 for the review. The leading term in the self-action force is proportional to a large factor $\left(\lambda_{0} / R\right)^{3}$ and can be interpreted as a non-retarded London-van-der-Waals interaction with an instantaneous image dipole. Here $\lambda_{0}=2 \pi c / \omega_{0}$ is the vacuum wavelength of a given dipole transition with frequency $\omega_{0}, \varepsilon_{1}$ is the dielectric constant of the medium in which an atom is located. This large factor originates from the near field

$$
\mathbf{E}_{\perp, \|}=-\frac{(\mp 3-1) \mathbf{p}_{\perp, \|}^{\prime}}{16 R^{3} \varepsilon_{1}}, \quad \mathbf{p}_{\perp, \|}^{\prime}=\mp \frac{\mathbf{p}_{\perp, \|}\left(\varepsilon_{1}-\varepsilon_{2}\right)}{\varepsilon_{1}+\varepsilon_{2}}
$$

created by a high-frequency image dipole $\mathbf{p}^{\prime}$ at the position of a real dipole $\mathbf{p}$. Here and below upper and lower signs correspond to the dipoles oriented perpendicular $(\perp)$ and parallel $(\|)$ to the boundary.

There is a number of reasons why the dynamics of molecular radiative transitions is modified in the presence of boundaries. The effects that were identified and observed include (i) changes in the spectral density of radiated modes [3, 7, 8, 9, 17], (ii) location of an atom in the nodes or maxima of resonant modes or in a non-transparent medium, e. g., in a medium with negative dielectric constant or in a photonic band structure, when the transition frequency is inside the Bragg gap 119, 20, 21, (iii) phase shift of the near field of a dipole due to dissipation in the neighboring medium 22, 23].

Additional possibilities to affect the molecular dynamics arise when the dipoles are moving near the interface of two media or inside a medium. A well-known example is Cherenkov radiation of an oscillating dipole moving in an anisotropic medium under the conditions of anomalous Doppler effect which can lead to instability of the dipole oscillations 24]. This instability is, however, very difficult to realize since radiation in the directions corresponding to the normal Doppler effect usually dominates.

In this paper we investigate a new mechanism of boundary-induced excitation of molecular transitions which is realized when a molecule is moving close to the dielectric or conducting medium with periodic grating. In this case the radiation reaction force acting on an oscillating dipole moment of a given transition is mainly due to the time-dependent London-van-der-Waals interaction experienced by the molecule moving above a grating. It is proportional to the instantaneous value of the dipole moment with a proportionality coefficient being a periodic function of time. Its modulation frequency 
$\nu$ is equal to the velocity of a molecule divided by the spatial period of a grating. Such a modulated radiation reaction force represents a periodic perturbation of the transition frequency $\omega_{0}$ (eigenfrequency of dipole oscillations) which can drive a parametric instability under the conditions of Mathieu's resonance: $\nu=2 \omega_{0} / N$, where $N$ is $1,2,3 \ldots$ In other words, the instability is due to the periodically modulated radiation reaction force which gives rise to excitation of dipole oscillations even from the level of quantum or thermal fluctuations.

If we have a beam of molecular dipoles, one can expect generation of measurable coherent radiation flux.

We emphasize that this situation is different from the well-known Smith-Purcell effect [25] in which a charge moving above a periodic grating creates an oscillating dipole due to its oscillating image. It is also different from the transition radiation of a charge traversing a periodic dielectric stack [26]. In the present case, it is the electrically neutral atom or molecule that gets parametrically excited.

To find the conditions for parametric instability, we calculate the back reaction force acting on a dipole oscillator with a high-frequency dipole moment $\mathbf{p}(t)$ which is moving with a constant velocity $v$ in a medium with real dielectric constant $\varepsilon_{1}$ near the boundary of a medium with complex dielectric constant $\varepsilon_{2}$. Precisely, we calculate the electric field $\mathbf{E}(t)$ created by a high-frequency dipole at its position $z=R, x=v t, y=0$. We assume that the medium 2 has a periodic grating with period $L$ in the direction $x$ along the dipole velocity, so that the distance $R$ between the dipole and the boundary is actually a periodic function of time with period $T=L / v$.

We assume that the distance $R$ to the boundary is much less than the grating period, so that the radiation reaction field at any moment of time $t$ is approximately equal to the one for the dipole at a distance $z(t)$ above an infinite plane boundary. This is of course the most interesting limit. In the opposite case, when $R \gg L$, the dipole will "feel" simultaneously many grating periods and the effect of grating will be averaged almost to zero.

The problem of the radiation reaction field acting on a dipole above a plane boundary was considered many times. We will use here the results of our papers [22, 23. According to these works, the radiation reaction field can be represented as a sum of a free-space term $\mathbf{E}_{\text {free }}(t)=$ $2 \dddot{\mathbf{p}}(t) \sqrt{\varepsilon_{1}} / 3 c^{3}$ and a boundary-induced term $\mathbf{E}_{b}$ that can be very complicated.

If the medium 2 is a perfect conductor, $\varepsilon_{2} \rightarrow i \infty$, then the contribution of a boundary is equivalent to the field of an image dipole $\mathbf{p}_{\|}^{\prime}=-\mathbf{p}_{\|}$or $\mathbf{p}_{\perp}^{\prime}=\mathbf{p}_{\perp}$, retarded by the time $t_{1}=2 R \sqrt{\varepsilon_{1}} / c$ :

$$
\mathbf{E}_{b \perp, \|}(t)=\frac{(1 \mp 1) \ddot{\mathbf{p}}_{\perp, \|}\left(t-t_{1}\right)}{4 R c^{2}}+\frac{\dot{\mathbf{p}}_{\perp, \|}\left(t-t_{1}\right)}{(3 \mp 1) R^{2} c \sqrt{\varepsilon_{1}}}
$$

$$
+\frac{\mathbf{p}_{\perp, \|}\left(t-t_{1}\right)}{2(3 \mp 1) R^{3} \varepsilon_{1}} .
$$

This result was obtained in many works, including the full quantum-electrodynamical treatment of the problem; see Refs. [1-5,13,15,17,19,22,23].

To illustrate the physical mechanism of an instability we will assume in this paper that the medium 2 is a perfect conductor and use the above expression (2) for the field. The case of an arbitrary medium is qualitatively similar and can be analyzed by using general expressions for the field obtained in 22, 23 .

Parametric instability of a harmonic dipole oscillator. This case is relevant for dipole transitions in a system with many quasiequidistant levels, for example, vibrational transitions in a molecule or Rydberg transitions in an excited atom. Such a system has essentially classical dynamics. The equation for free dipole oscillations of a classical harmonic oscillator with a charge $e$, mass $m$, and frequency $\omega_{0}$ takes the form

$$
d^{2} \mathbf{p} / d t^{2}+\omega_{0}^{2} \mathbf{p}=\left(e^{2} / m\right)\left(\mathbf{E}_{\text {free }}+\mathbf{E}_{b}\right) .
$$

In the free space, this equation describes an oscillator decaying with radiative rate $\gamma=2 e^{2} \omega_{0}^{2} \sqrt{\varepsilon_{1}} /\left(3 m c^{3}\right)$.

In the presence of a boundary, let us consider for definiteness the dipole oriented perpendicular to the interface between two media. The result for a parallel dipole is the same apart from a different numerical coefficient. Then, expanding the electric field $\mathbf{E}_{b \perp}$ in Eq. (2) in powers of a small parameter $R / \lambda_{0}$, we obtain the following equation:

$$
\ddot{p}+2 \gamma \dot{p}+\left(\omega_{0}^{2}-\frac{e^{2}}{4 m R^{3} \varepsilon_{1}}\right) p=0 .
$$

The factor $2 \gamma$ describes the well-known result that a perpendicular dipole above the metal surface decays with a rate twice that in a free space. Our main interest here is in the frequency shift factor which is proportional to $1 / R^{3}$ and can have much larger value than radiative decay proportional to $1 / \lambda^{3}$. Note that for an arbitrary medium 2 with complex dielectric constant $\varepsilon_{2}$ this frequency shift has also imaginary part $\propto \varepsilon_{2}^{\prime \prime} / R^{3}$ that can be larger than $\gamma$. This can lead to strong modification of the spontaneous emission rate and even reversal of the direction of radiative transitions [22, 23].

For an ideal conductor the frequency shift is real, and the only possible mechanism of instability is parametric excitation of dipole oscillation due to periodic resonance modulation of this term. This can be achieved by, e.g., modulating the distance $R$ to the boundary. Such a modulation occurs when, for example, the molecule is moving above a periodic grating of period L. Note that we consider here free dipole oscillations, without any external electromagnetic field force.

Suppose for simplicity that the grating is sinusoidal. Then the distance $R$ in the expression for the radiation 
reaction field will be periodically modulated as

$$
R=R_{0}(1+a \cos \nu t),
$$

where $\nu=2 \pi v / L$. The parametric resonance occurs when $\nu=2 \omega_{0} / N$, for an integer $N=1,2, \ldots$.

If the relative grating amplitude is small, $a \ll 1$, we obtain from Eq. (何) the Mathieu's equation:

$$
\ddot{p}+2 \gamma \dot{p}+\omega_{0}^{2}(1+A \cos \nu t) p=0,
$$

where

$$
A=\frac{3 e^{2} a}{4 m R_{0}^{3} \omega_{0}^{2} \varepsilon_{1}} .
$$

The condition for instability on the main resonance $N=$ 1 reads $A>4 \gamma / \omega_{0}$, which can be rewritten as

$$
\frac{9}{256 \pi^{3} \varepsilon_{1}^{3 / 2}}\left(\frac{\lambda_{0}}{R_{0}}\right)^{3} a>1 .
$$

Assuming $a=0.1$, the numerical factor on the left-hand side of (7) is of order $10^{-4}$, which requires $\lambda_{0} / R_{0}>20$. Since $R_{0}$ cannot be reasonably made much smaller than $0.1 \mu \mathrm{m}$, we need to use long-wavelength transitions, e.g., vibrational-rotational transitions in molecules.

In addition, the resonance condition implies $\lambda_{0}=$ $2 c L / v$. The value of $L$ is bounded from below: $L>R_{0} \gtrsim$ $0.1 \mu \mathrm{m}$. The velocities of neutral molecules achieved in supersonic jets or Laval nozzles are of the order of 1 $\mathrm{km} / \mathrm{s}$. In this case $\lambda$ falls into the $\mathrm{cm}$ range corresponding to rotational transitions that are better described by a two-level model (see below). However, by accelerating charged molecules and then neutralizing them much greater velocities can be achieved that allow one to employ IR and sub-mm vibrational transitions with quasiequidistant energy levels.

Not too close to the threshold, the growth rate of dipole oscillations is

$$
\omega^{\prime \prime} \simeq \omega_{0} A / 4 \simeq 3 e^{2} a /\left(16 m \omega_{0} R_{0}^{3} \varepsilon_{1}\right) .
$$

Using the correspondence principle we can relate the classical dipole amplitude with the matrix element $d$ of a given dipole transition: $e^{2} / m=2 \omega_{0} d^{2} / \hbar$. Then Eq. (8) yields the growth rate

$$
\omega^{\prime \prime}=3 d^{2} a /\left(8 \hbar R_{0}^{3} \varepsilon_{1}\right) .
$$

For a typical dipole moment $d \sim 1$ Debye and $R_{0} \sim 0.1$ $\mu \mathrm{m}$, we get $\omega^{\prime \prime} \sim 5 \times 10^{4} \mathrm{~s}^{-1}$, which means that an excitation length $v / \omega^{\prime \prime}$ is $2 \mathrm{~cm}$. Therefore, the excitation length is short enough to make the effect readily observable in experiment.

Instability of a two-level system. An opposite limiting case of a molecular transition is a two-level system.
A good example in the low frequency range is rotational transitions in molecules. One can obtain the modified Bloch equations for a two-level molecule in the vicinity of an interface following the usual derivation of master equations for a two-level system in a free space. The result is presented below, again for a perpendicular dipole:

$$
\begin{aligned}
\ddot{\mathbf{p}}+2 \gamma \dot{\mathbf{p}}+\left(\omega_{0}^{2}\right. & \left.-d^{2} \omega_{0} \Delta n /\left(2 \hbar R^{3}\right)\right) \mathbf{p} \\
& =2 \Delta n d^{2} \omega_{0} \hbar^{-1} \mathbf{E}_{\text {ext }}, \\
d(\Delta n) / d t+\gamma\left(\Delta n-\Delta n_{p}\right) & =-\left(2 / \hbar \omega_{0}\right) \mathbf{E}_{\text {ext }} \dot{\mathbf{p}} .
\end{aligned}
$$

Here $\Delta n=n_{1}-n_{2}$ is volume density of the population difference between two states, $\Delta n_{p}$ is the population difference supported by an incoherent pumping, $\mathbf{E}_{\text {ext }}$ is an external (classical) field which does not contain any backreaction field.

In the absence of an external field we obtain the growth rate of the parametric instability,

$$
\omega^{\prime \prime}=\frac{3 d^{2} a \Delta n}{8 \hbar R_{0}^{3} \varepsilon_{1}},
$$

which is different from Eq. (9) only by a factor $\Delta n$. This factor can be rather small for the rotational transitions at room temperature. This may require low temperatures for more efficient excitation. To reach $\Delta n \sim 1 / 2$ for $\omega_{0}=10^{11} \mathrm{~s}^{-1}$, liquid helium temperatures are required.

The value of the growth rate can be enhanced even further by launching molecules closer to the surface or decreasing the dielectric constant $\varepsilon_{1}$. It is difficult to send molecules closer than $0.1 \mu \mathrm{m}$ to the surface. At the same time, the dielectric constant can be drawn to very small values by creating a rarefied background plasma with plasma frequency close to the frequency $\omega_{0}$ of dipole oscillations. For $\omega_{0}=10^{11} \mathrm{~s}^{-1}$ the density of ionized gas should be around $10^{12} \mathrm{~cm}^{-3}$. The plasma of such density can be very easily produced in a gas discharge. Collisions in such a rarefied plasma are unimportant, and the residual value of $\varepsilon_{1}$ will be defined by density close to the grating surface, where the double electric layer is formed. Experiments indicate the possibility to decrease $\varepsilon_{1}$ down to $10^{-4}$.

Such an enhancement of the parametric instability in a rarefied plasma is interesting by itself. The experimental realization of this effect is facilitated by the fact that fluctuations in $\varepsilon_{1}$ are not important since the quantity $\varepsilon_{1}$ in all expressions for the growth rate is actually the value averaged over a very large spatial scale $\lambda_{1}=\lambda_{0} / \sqrt{\varepsilon_{1}}$.

Possible experiments. There is a number of ways how this unusual dynamics of a molecule can be observed. One evident possibility is to detect radiation due to excited dipole oscillations from a beam of molecules flying above the grating. The density $n$ in a molecular beam moving with supersonic velocity in a supersonic jet or Laval nozzle can be as high as $10^{17} \mathrm{~cm}^{-3}$. However, since only the molecules moving very close to the 
surface ( $L \leq 0.1 \mu \mathrm{m}$ in our numerical example) are efficiently excited, the total amount of excited molecules above the grating plate of $1 \mathrm{~cm} \times 10 \mathrm{~cm}$ size will be of order $N \sim 10^{13}$. Each molecule radiates with a power of order $W_{1} \simeq \gamma \hbar \omega_{0}$. At a frequency $\omega_{0}=10^{11} \mathrm{~s}^{-1}$ their total spontaneous emission power will be very small: $N W_{1} \sim 10^{-16} \mathrm{~W}$. To increase the radiated power, one should pre-phase the dipole oscillations of molecules at the entrance to the amplification region by an external microwave field of intensity higher than the thermal noise. Then the molecules will radiate coherently in the bunches of length of the order of wawelength $\lambda$, and the power radiated by one bunch will be $N^{2}$ times the power radiated by a single molecule, where $N \sim n \lambda^{2} R_{0}$. For the above values of $n$ and $\lambda \sim 1 \mathrm{~cm}$ we obtain the total power in the microwatt range which is easily detectable.

Another way to study the dynamics of molecules is to observe the change in absorption of a microwave radiation propagating through the layer of parametrically excited molecules. This scheme is also easily realizable.

To increase further the radiated power and to facilitate the requirements for experiments it is desirable to launch molecules with higher speed. This increases the frequency of parametric modulation and leads to greater power of spontaneous emission (which goes as $\omega^{4}$ ). Then one can excite vibrational transitions in molecules and obtain an emitter in the far-IR range where very few sources exist. Alternatively, for a given resonant frequency we can increase the spatial period $L$ of the grating and the distance $R$ from the interface. However, it is difficult to accelerate neutral molecules to velocities higher than $1 \mathrm{~km} / \mathrm{s}$. A way to overcome this difficulty is to accelerate molecular ions and then neutralize them by charge exchange. This can be achieved by putting a buffer gas cell or a thin foil before the entrance to the excitation region with grating interface.

We conclude that there is one more fundamental effect in the realm of QED, namely, the parametric selfexcitation of dipole oscillations of a molecule moving close to a periodic grating. This effect can be experimentaly observed in different ways and can be even employed for generation of coherent radiation in the far-IR to microwave ranges.

Acknowledgments. We thank Marlan Scully for valuable discussions and appreciate the support from the Texas Advanced Technology Program, the Office of Naval research, and High Energy Lasers Program of Department of Defense.

[1] K.H. Drexhage, in Progress in Optics, edited by E. Wolf, 12, 163 (1974).
[2] R.R. Chance, A. Prock, and R. Silbey, Adv. Chem. Phys. 37, 1 (1978);

J.M. Wylie, J.E. Sipe, Phys. Rev. A 30, 1185 (1984); Ibid. A 32, 2030 (1985).

[3] E.A. Hinds, Adv. Atom. Mol. Opt. Phys. 28, 237 (1991); Adv. Atom. Mol. Opt. Phys. Suppl. 2, 1 (1994).

[4] H. Morawitz, Phys. Rev. 187, 1792 (1969); P.W. Milonni, P.L. Knight, Opt. Comm. 9, 119 (1973).

[5] H.F. Arnoldus, T.F. George, Phys. Rev. A 37, 761 (1988).

[6] H. Khosravi, R. Loudon, Proc. Roy. Soc. Lond. A 433, 337 (1991); Ibid. A 436, 373 (1992);

S.M. Barnett, B. Huttner, and R. Loudon, Phys. Rev. Lett. 68, 3698 (1992).

[7] A.N. Oraevsky, Usp. Fiz. Nauk 164, 415 (1994).

[8] V.F. Cheltsov, Nuovo Cim. D 17, 17 (1995).

[9] D. Meschede, Phys. Rep. 211, 201 (1992).

[10] Y. Yamamoto, S. Machida, and G. Bjork, Opt. Quant. Electr. 24, 215 (1992).

[11] H. Casimir, D. Polder, Phys. Rev. 73, 360 (1948).

[12] G. Barton, Proc. Roy. Soc. Lond. 320, 251 (1970); Ibid. A 410, 141, 175 (1987); J. Phys. B 7, 2134 (1974).

[13] P.W. Milonni, Phys. Rev. A 25, 1315 (1982); Phys. Scripta T 21, 102 (1988).

[14] J. Dalibard, J. Dupont-Roc, and C. Cohen-Tannoudji, J. de Physique 43, 1617 (1982); Ibid. 45, 637 (1984).

[15] D. Meschede, W. Jhe, and E.A. Hinds, Phys. Rev. A 41, 1587 (1990).

[16] E.A. Hinds, V. Sandoghdar, Phys. Rev. A 43, 398 (1991).

[17] A.O. Barut, J.P. Dowling, Phys. Rev. A 36, 649 (1987).

[18] G.S. Agarwal, Phys. Rev. A 12, 1475 (1975);

G.S. Agarwal, S.D. Gupta, R.R. Puri, Fundamentals of cavity quantum electrodynamics, World Scientific, Singapore (1995);

H. Morawitz, M.R. Philpott, Phys. Rev. B 10, 4863 (1974).

[19] V.P. Bykov, Zh. Eksp. Teor. Fiz. 62, 505 (1972), Ibid. 63, 1226 (1972) (Sov. Phys. - JETP 36, 646 (1973)); Kvant. Elektron. (Moscow) 1, 1557 (1974) (Sov. J. Quant. Electron. 4, 861 (1975)).

[20] S. John, T. Quang, Phys. Rev. A 50, 1764 (1994).

[21] T. Suzuki, P.K.L. Yu, J. Opt. Soc. Am. B 12, 570 (1995); M. Scalora, J.P. Dowling, M. Tossi, M.J. Bloemer, C.M. Bowden, J.W. Haus, Appl. Phys. B, 60, S57 (1995). .

[22] A.A. Belyanin, V. V. Kocharovsky, and Vl.V. Kocharovsky, Laser Physics 5, 1164 (1995).

[23] V.V. Kocharovsky, Vl.V. Kocharovsky, and A.A. Belyanin, Phys. Rev. Lett. 76, 3285-3288 (1996).

[24] V.L. Ginzburg and V.M. Fain, JETP 35, 817 (1958).

[25] S.J. Smith and E.M. Purcell, Phys. Rev. 92, 1069 (1953).

[26] V.L. Ginzburg and I.M. Frank, JETP 16, 15 (1946). 